\title{
Modelo de atenuação por vegetação em bosques urbanos na faixa UHF
}

\author{
Maurício H. C. Dias ${ }^{1}$ e Mauro S. Assis ${ }^{2}$
}

\begin{abstract}
Resumo-A atenuação por vegetação é um fator relevante para predição de cobertura de sistemas-rádio em frequências elevadas. Modelos empíricos são uma abordagem numérica vantajosa para estimar este tipo de perda. Este trabalho apresenta um modelo de perda por vegetação baseado em medidas realizadas em um bosque urbano na cidade do Rio de Janeiro, na faixa de 0,9 a $1,8 \mathrm{GHz}$. A expressão resultante tem a forma indicada na Recomendação P.833 do UIT-R, e contribui com parâmetros que podem ser aplicados em cenários similares ao deste trabalho. Outros modelos empíricos foram testados e avaliados quanto à aderência estatística aos dados.
\end{abstract}

Palavras-Chave-Propagação de ondas, modelos de propagação, atenuação por vegetação, sistemas UHF.

Abstract-Vegetation attenuation is a relevant issue for radio systems coverage prediction at high frequencies. Empirical modeling is a numerical attractive approach to estimate this type of loss. This work presents a vegetation loss model derived from measurements performed inside a woodland at Rio de Janeiro city, from 0.9 to $1.8 \mathrm{GHz}$. The resulting equation has the same general terms indicated in the ITU-R Recommendation P.833, and contributes with parameters that can be useful in similar application scenarios. Other empirical models have been tested and assessed regarding statistical adherence to the available data.

Index Terms-Radiowave propagation, propagation modeling, vegetation attenuation, UHF systems.

\section{INTRODUÇÃO}

No contexto das telecomunicações sem fio, uma das atividades necessárias para o adequado planejamento de sistemas é a predição de cobertura. Para tal, é preciso dispor de informações sobre o cenário onde o sistema será operado e escolher modelos apropriados de estimação da perda média ou mediana de percurso do sinal eletromagnético. A classe de modelos mais simples e de menor demanda numérica é a dos modelos empíricos ou semi-empíricos, que expressam a perda em função de alguns poucos parâmetros do sistema e do ambiente [1]. Um exemplo clássico é o modelo de OkumuraHata, em que a perda é função da distância, da frequência, das alturas efetivas das antenas e da morfologia do terreno [2].

\footnotetext{
1 Seção de Engenharia Elétrica, Instituto Militar de Engenharia, Rio de Janeiro, Brasil. E-mail: mhcdias@ime.eb.br.

2 Departamento de Engenharia de Telecomunicações, Universidade Federal Fluminense, Niterói, Brasil: E-mail: msassis@ openlink.com.br
}

A abordagem de predição de cobertura baseada no uso de modelos semi-empíricos é usualmente complementada por ajustes pontuais para tentar cobrir situações especiais que ocorrem com razoável frequência no cenário de interesse, mas que estão fora do escopo do modelo adotado. A difração em obstáculos elevados ao longo do terreno é um exemplo recorrente em sistemas pessoais sem fio operando em ambientes urbanos. O efeito da vegetação presente nestes mesmos cenários (árvores principalmente) é outro aspecto que costuma merecer tratamento adicional no planejamento de sistemas móveis [3]-[4].

Os três tipos de disposição da vegetação em meios urbanos que merecem maior atenção na modelagem da perda de propagação associada são: árvores isoladas; fileiras de árvores ao longo das ruas; e parques ou bosques. As abordagens usuais para estudar os efeitos da vegetação, por sua vez, são teóricas ou baseadas em medidas [5].

Uma das abordagens teóricas para expressar os efeitos da vegetação é a teoria de espalhamento discreto de Foldy e Lax [6]-[8], em que as árvores são modeladas como aglomerados de espalhadores discretos (discos e cilindros, representando folhas e troncos, respectivamente) distribuídos aleatoriamente de maneira esparsa. Exemplos da aplicação e validação por medidas desta modelagem podem ser encontrados em [9], que avaliou o efeito de fileiras de árvores ao longo das ruas em ambientes suburbanos, e em [10]-[11], que estudaram a perda adicional devida à passagem da onda eletromagnética por bosques urbanos. Outra abordagem teórica pertinente se baseia na teoria da transferência de energia por radiação (RET - Radiative Energy Transfer), utilizada em [12]-[13] como base para modelar a perda de propagação em árvores isoladas e em bosques

As abordagens experimentais são mais difíceis de generalizar e não traduzem de forma pormenorizada os complexos mecanismos de propagação envolvidos. Entretanto, os modelos baseados em medidas apresentam expressões analíticas fechadas, de aplicação imediata, atributo desejável em modelos de predição. Exemplos de modelos empíricos para a atenuação devida a árvores isoladas podem ser encontrados em [14]-[15]. A atenuação em parques urbanos, por sua vez, é analisada a partir de medidas de campo em [16]-[17].

Uma importante referência sobre a atenuação por vegetação é a Recomendação P.833 do UIT-R (Setor de Radiocomunicações da UIT) [18], que cobre sinais entre $30 \mathrm{MHz}$ e $60 \mathrm{GHz}$. Este documento é composto por contribuições de diversos pesquisadores dos países membros 
da UIT (União Internacional de Telecomunicações), contendo modelos para o cálculo da perda e considerações técnicas gerais sobre o tema. O modelo teórico baseado em RET de [13], por exemplo, foi incluído em [18] como solução para o problema da obstrução de uma árvore isolada interposta entre dois terminais. Por outro lado, para o problema da atenuação por vegetação quando um dos terminais está dentro de uma área arborizada (parques, bosques ou florestas), a solução indicada é a modelagem empírica, embora a própria Recomendação P.833 disponha de apenas algumas expressões completas representativas de alguns poucos cenários. Uma dessas expressões foi obtida a partir de uma campanha de medidas realizada em um parque no Centro da cidade do Rio de Janeiro, cobrindo a faixa de frequências de 900 a $1800 \mathrm{MHz}$, da qual os autores participaram [5]. O presente trabalho descreve em detalhes esta campanha de medidas e o modelo de perda por vegetação resultante. O texto apresenta, ainda, uma análise comparativa da expressão proposta com outros dois modelos empíricos disponíveis na literatura.

Este artigo foi estruturado da seguinte forma. A seção II trata de modelos da atenuação por vegetação em bosques urbanos, com foco na abordagem recomendada pelo UIT-R em [18]. O modelo proposto, por sua vez, é apresentado na seção III, que descreve a campanha de medidas e a metodologia de ajuste dos dados obtidos à forma da expressão geral de [18]. A análise comparativa das medidas com o modelo proposto e com outros modelos disponíveis é o foco da seção IV. Por fim, a seção V conclui o artigo.

\section{Modelos DA ATENUAÇÃo POR VEGETAÇÃo EM BOSQUES URBANOS}

Em [10], um modelo teórico é proposto para o cálculo da atenuação específica correspondente à propagação através de florestas ou bosques, na faixa de 0,1 a $10 \mathrm{GHz}$. A base do modelo foi a teoria de espalhamento discreto de Foldy e Lax [6]-[8]. Entretanto, como as expressões desta teoria não têm forma fechada, o modelo proposto incorporou simplificações decorrentes da análise de dados estatísticos e características elétricas da folhagem estudada, bem como de resultados obtidos por medidas. Abordagens similares foram adotadas em [11] e [19] para o mesmo tipo de disposição de vegetação.

O modelo teórico apresentado em [13], incorporado à Recomendação P.833 [18], apresenta expressões para o cálculo da perda adicional de propagação em um enlace obstruído por uma árvore isolada. A perda é modelada como a soma de três contribuições associadas aos seguintes mecanismos de propagação: difração nas laterais e na copa da árvore; reflexão no solo; e espalhamento ou transmissão através da árvore. Expressões analíticas fechadas são utilizadas para os cálculos das duas primeiras parcelas. A perda por espalhamento é dada por expressão iterativa derivada da teoria da transferência de energia por radiação. A expressão final depende de muitos parâmetros geométricos e elétricos relacionados ao enlace e à árvore. Os autores realizaram diversas medidas para gerar um banco de parâmetros representativos de várias espécies de árvores típicas de regiões temperadas do hemisfério Norte, disponibilizado em [18]. Cumpre destacar que apesar de representar a perda em uma única árvore, o modelo pode ser empregado iterativamente para calcular a contribuição de um número qualquer de árvores dispostas de qualquer maneira em uma determinada região, se as posições das árvores forem especificadas, como exemplificado pelos próprios autores em [13].

Um modelo empírico para a faixa de 0,2 a $95 \mathrm{GHz}$ indicado em um Relatório [20] do ex-CCIR (Comitê Consultivo Internacional de Rádio), atual UIT-R, ainda é bastante referenciado na literatura, mesmo em trabalhos mais recentes. A atenuação adicional por vegetação $A_{v}(\mathrm{~dB})$ é dada por:

$$
A_{v}=0,2 \cdot f^{0,3} \cdot d^{0,6}
$$

onde $f$ é a frequência $(\mathrm{MHz})$ e $d(\mathrm{~m})$ a distância percorrida pelo sinal na vegetação.

A Recomendação atual do UIT-R sobre perda por vegetação, P.833 [18], divide o tema em cinco grandes tópicos: perda em enlaces com um terminal dentro da área com vegetação (bosques e florestas); obstrução de árvore isolada; despolarização; efeitos dinâmicos; e características de espalhamento de retardos. A expressão geral indicada para a perda adicional $A_{v}(\mathrm{~dB})$ devida à propagação através de trechos contínuos de vegetação é dada por:

$$
A_{v}=A_{m}\left(1-e^{-\gamma \cdot d / A_{m}}\right)
$$

onde $d(\mathrm{~m})$ é a distância atravessada pelo sinal na vegetação, $\gamma$ é a atenuação específica para trechos de vegetação muito curtos $(\mathrm{dB} / \mathrm{m})$, e $A_{m}$ é a atenuação máxima para um terminal dentro de uma floresta específica $(\mathrm{dB})$. A Fig. 1 ilustra o comportamento típico correspondente de variação da perda com a distância.

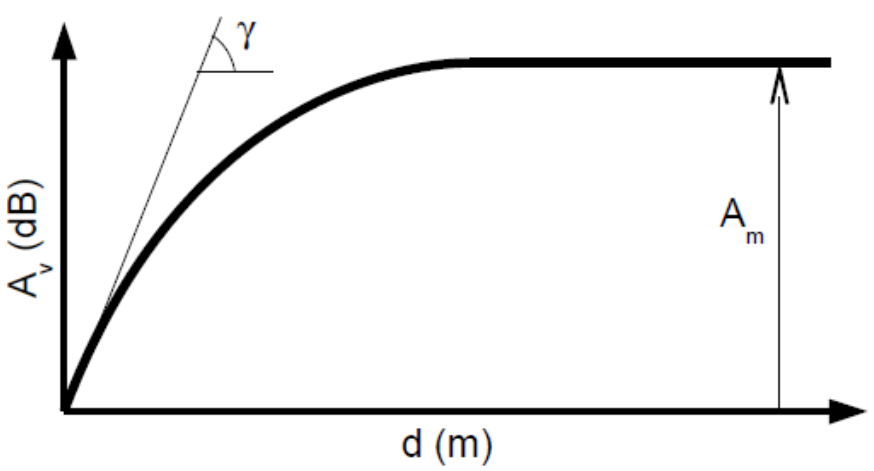

Fig. 1. Forma geral da perda adicional por vegetação indicada em [18].

Valores típicos para a atenuação específica $\gamma$ na faixa de $30 \mathrm{MHz}$ e $60 \mathrm{GHz}$ são indicados em [18], para as polarizações vertical e horizontal. A recomendação não traz, entretanto, valores gerais para $A_{m}$. Embora a forma geral representada por (2) esteja presente na P.833 desde sua primeira versão de 1992, até hoje há poucos trabalhos que buscam identificar parâmetros $A_{m}$ para diferentes tipos de vegetação, em diferentes frequências. $\mathrm{Na}$ versão atual da P.833, constam apenas duas contribuições distintas baseadas em medidas para este parâmetro, ambas com a forma: 


$$
A_{m}=A_{1} f^{\alpha}
$$

onde $A_{1}(\mathrm{~dB})$ e $\alpha$ são obtidas a partir do ajuste dos dados a expressão em (3), e a frequência $f$ é dada usualmente em MHz. Uma das contribuições foi baseada em medidas em bosques na França, contendo várias espécies nativas, com altura média das copas de árvores igual a $15 \mathrm{~m}$, na faixa de 0,9 a 2,3 GHz. Para esta campanha de medidas, $A_{1}=1,15$ e $\alpha=0,43$. A outra contribuição corresponde ao modelo resultante das medidas descritas neste artigo, detalhado na próxima seção.

Em uma das poucas referências com análises de desempenho do modelo atual do UIT-R representado por (2), a atenuação por vegetação foi avaliada com base em medidas tomadas para distâncias de no máximo $45 \mathrm{~m}$ em pequenos bosques na Grã-Bretanha, na frequência de $38 \mathrm{GHz}$ [17]. A aderência de (1) aos dados foi insatisfatória, o que levou os autores a testarem uma versão modificada de (1), ajustada aos dados disponíveis, dada por:

$$
A_{v}=0,37 \cdot f^{0,3} \cdot d^{0,38}
$$

Os conjuntos de dados foram ajustados a (2) também, com aderência equivalente à obtida com (4).

\section{MODElo EXPERIMENTAL PROPOSTO}

\section{A. Descrição da Campanha de Medidas}

O local de realização das medidas foi o Campo de Santana, um pequeno parque de cerca de $320 \mathrm{~m} \times 520 \mathrm{~m}$ na região central do Rio de Janeiro, com grande concentração arbórea ao longo de quase toda sua área. $\mathrm{O}$ sinal foi transmitido do Palácio Duque de Caxias (PDC), um prédio com 22 andares, situado em frente a uma das entradas do parque. O terreno da região analisada é plano, sem irregularidades topográficas. A Fig. 2 ilustra o cenário em questão.

No Campo de Santana há uma clara predominância de uma espécie de árvore, conhecida por Ficus Religiosa. Esta espécie apresenta alturas típicas da ordem de $15 \mathrm{~m}$, troncos com cerca de 2 a $3 \mathrm{~m}$ de diâmetro, galhos e ramos com mais de $5 \mathrm{~m}$ de extensão e diâmetros variados, de dezenas até centenas de mm. Suas folhas são finas (espessura $<1 / 2 \mathrm{~mm}$ ) e pequenas (3-5 cm de diâmetro) e suas copas são, grosso modo, semi-esféricas ou semi-elípticas, com diâmetro (ou eixo horizontal maior) da ordem de $10 \mathrm{~m}$. O parque contém ainda pequenos lagos, vegetação rasteira, algumas poucas construções de pequeno porte e é rodeado por diversos prédios, alguns dos quais com alturas bem maiores que as das árvores.

Para realização das medidas, um gerador de sinais conduzia um sinal faixa estreita (tom) não modulado para uma antena de transmissão posicionada na janela de determinados andares do PDC frontal ao parque. $\mathrm{O}$ sinal propagado sensibilizava a antena de recepção, situada no topo de uma viatura do tipo furgão (a 2,4 m de altura), passava por um amplificador de baixo ruído (LNA) e era recebido por um analisador de espectro, dentro da viatura, que permanecia parada para o registro da medida. Com o analisador em modo span zero sintonizado na frequência de medição, a saída de tensão do analisador era gravada em fita magnética, por períodos de cerca de $30 \mathrm{~s}$. Posteriormente, o conjunto de dados coletados em campo era digitalizado para pós-processamento e análise, com taxa de aquisição alta o suficiente (até $500 \mathrm{~Hz}$ ) para acompanhar as flutuações rápidas do nível do sinal nas condições adotadas.

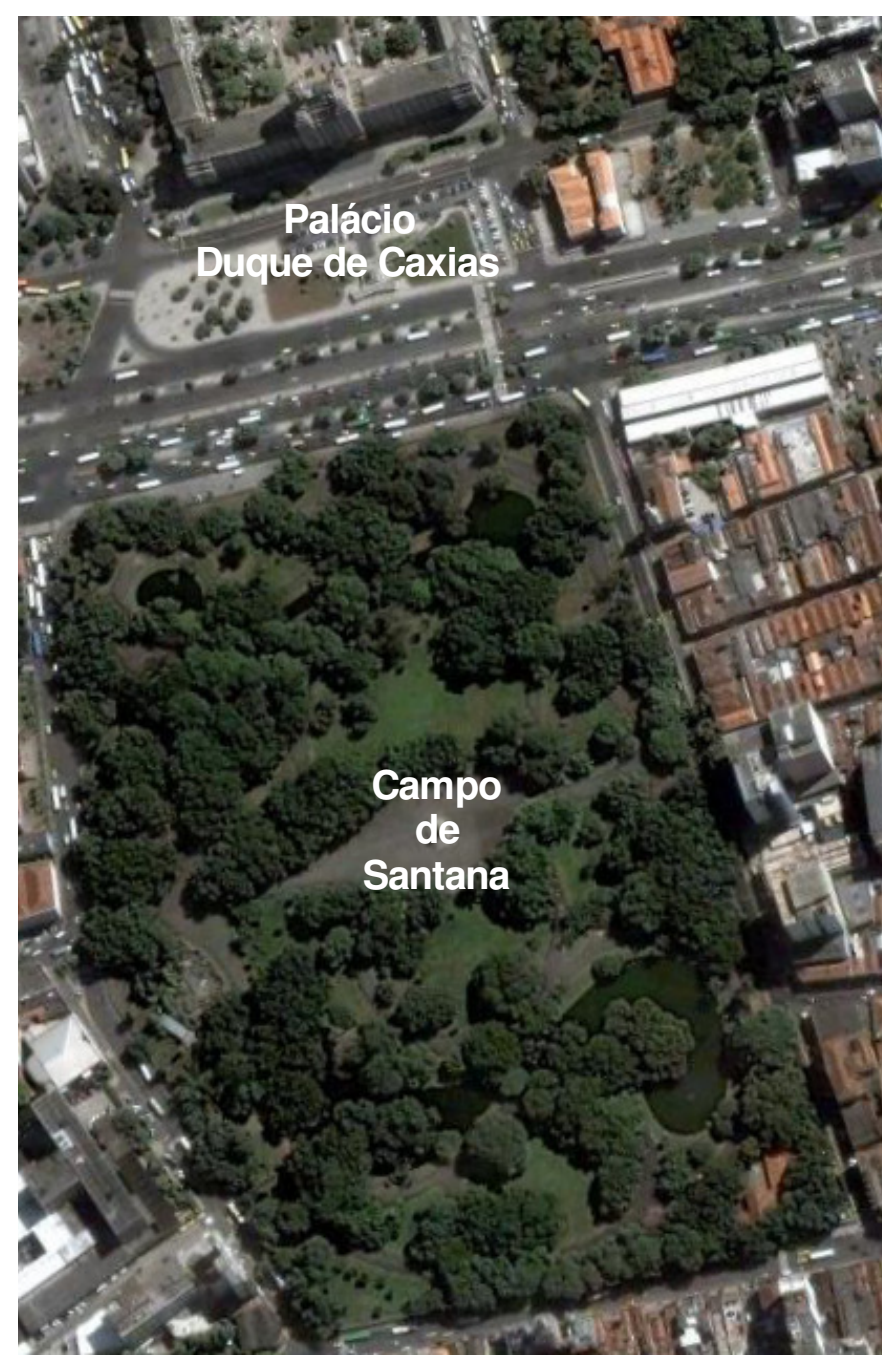

Fig. 2. Cenário da campanha de medidas.

A campanha foi realizada em duas etapas. A primeira consistiu de medidas em $0,9 \mathrm{GHz}$, em polarização horizontal e vertical. A segunda contemplou as frequências de 1,2, 1,5 e $1,8 \mathrm{GHz}$, apenas na polarização vertical. Todos os experimentos foram realizados em meses nos quais as árvores apresentavam folhagem completa. No Rio de Janeiro as diferenças entre as estações climáticas são pouco marcantes, e a época da queda das folhas representa apenas um período muito curto do inverno. Em ambas as etapas, a transmissão foi realizada a partir de sete andares distintos distribuídos ao longo dos 22 andares do PDC, todos com visada livre para o Campo de Santana, e a recepção foi registrada em 16 pontos diferentes dentro do parque. A potência na entrada da antena 
de transmissão era de $1 \mathrm{~W}$. Na primeira etapa, antenas dipolo de $1 / 2$ onda sintonizadas em $900 \mathrm{MHz}$ foram utilizadas tanto na transmissão quanto na recepção, e o ganho do LNA era de $30 \mathrm{~dB}$. Na segunda etapa, um arranjo log-periódico de dipolos foi usado na transmissão, com direção de apontamento para o centro do parque, enquanto a recepção foi feita com monopolos de $1 / 4$ de onda sobre plano de terra, sintonizados para cada uma das três frequências avaliadas.

Cumpre destacar que os dados obtidos da campanha de medidas em questão foram analisados também em outro trabalho, cujo foco principal foi a caracterização estatística das variações da resposta do canal de propagação associado [21].

\section{B. Ajuste dos Dados à Formulação Geral da ITU-R}

Com o foco do trabalho na modelagem da perda por vegetação, a primeira etapa do processamento dos dados foi a obtenção das médias (no tempo) das intensidades de sinal registradas. Em seguida, foram identificadas as distâncias totais envolvidas nos experimentos, bem como as respectivas distâncias efetivamente percorridas através da vegetação. Neste trabalho, estas distâncias foram determinadas a partir de um modelamento simplificado da geometria associada ao parque, que foi considerada essencialmente como um bloco retangular, cujas dimensões horizontais coincidiam com as do contorno externo do campo, e cuja altura era igual ao valor médio observado para as árvores do bosque $(15 \mathrm{~m})$. A interseção do raio direto entre o transmissor e o receptor com o bloco modelado foi considerada como a distância efetivamente atravessada pelo sinal dentro da vegetação (distância efetiva - d). A Fig. 3 ilustra a geometria assumida.

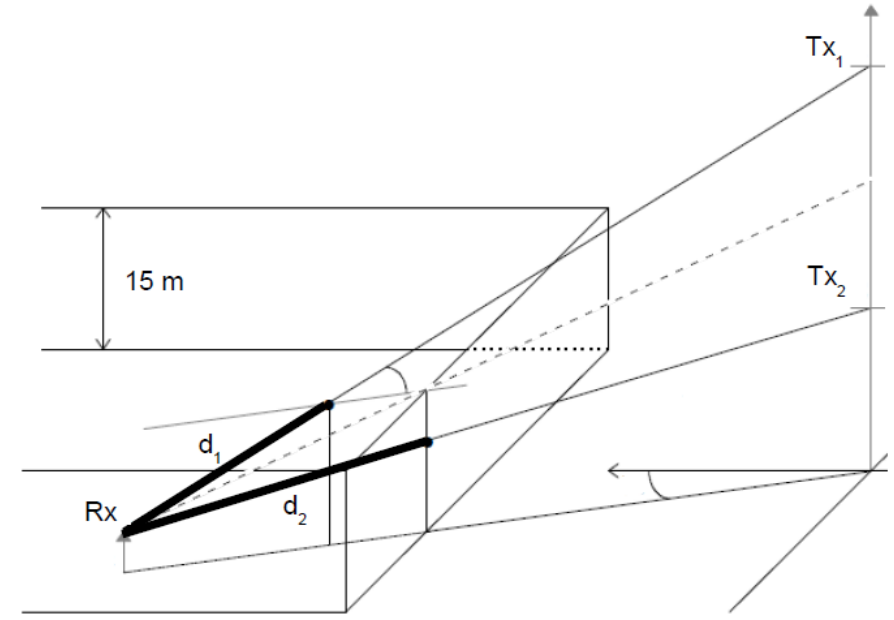

Fig. 3. Geometria adotada para estimar as distâncias efetivas.

De posse dos valores estimados de distâncias totais, dos dados de configuração dos enlaces e dos valores médios de potência recebida para cada enlace, calcularam-se os valores de atenuação no espaço livre correspondentes. Considerandose que a atenuação adicional sofrida pelos sinais medidos foi causada essencialmente pela vegetação, para cada enlace considerado, a atenuação por vegetação foi obtida da diferença entre a perda média total e a atenuação no espaço livre correspondente.
Para cada um dos cinco conjuntos de valores de atenuação por vegetação e das distâncias efetivas correspondentes, foi feito um ajuste dos dados a (2). A Tabela I apresenta os valores obtidos dos parâmetros $A_{m}$ e $\gamma$ para cada conjunto.

TABELA I

PARÂMETROS OBTIDOS POR AJUSTE DOS DADOS A (2)

\begin{tabular}{|c|c|c|c|}
\hline Frequência $(\mathrm{GHz})$ & Polarização & $A_{m}(\mathrm{~dB})$ & $\gamma(\mathrm{dB} / \mathrm{m})$ \\
\hline 0,9 & Horizontal & 29 & 0,3 \\
0,9 & Vertical & 31 & 0,2 \\
1,2 & Vertical & 32,3 & 0,5 \\
1,5 & Vertical & 53,5 & 0,2 \\
1,8 & Vertical & 46,5 & 0,4 \\
\hline
\end{tabular}

A obtenção de uma única expressão para todo o conjunto de dados passou por mais dois ajustes. Considerando apenas os parâmetros dos conjuntos de dados em polarização vertical, os quatro parâmetros respectivos da Tabela I foram ajustados a (3). Analogamente, os quatro coeficientes de atenuação específica da Tabela I foram ajustados a uma equação com a mesma forma de (3), ou seja, com dependência exponencial com a frequência. Os valores ajustados resultantes foram:

$$
\begin{aligned}
& A_{m}=0,18 f^{0,752} \\
& \gamma=0,0063 f^{0,537}
\end{aligned}
$$

\section{ANÁlise dos RESUltados}

A expressão de perda adicional por vegetação obtida a partir do ajuste dos dados a (2), representada pelos parâmetros em (5) e (6), foi comparada com outros modelos empíricos pertinentes. Em particular, foram considerados o modelo do ex-CCIR expresso por (1) e o modelo modificado de Seville, expresso por (4). As Figs. 4 a 8 apresentam scatter plots das atenuações médias por vegetação obtidas em comparação com os três modelos considerados, para os cinco conjuntos disponíveis. As tabelas II e III trazem as estatísticas de erros entre as curvas e os dados (erros médios e desviospadrão, respectivamente).

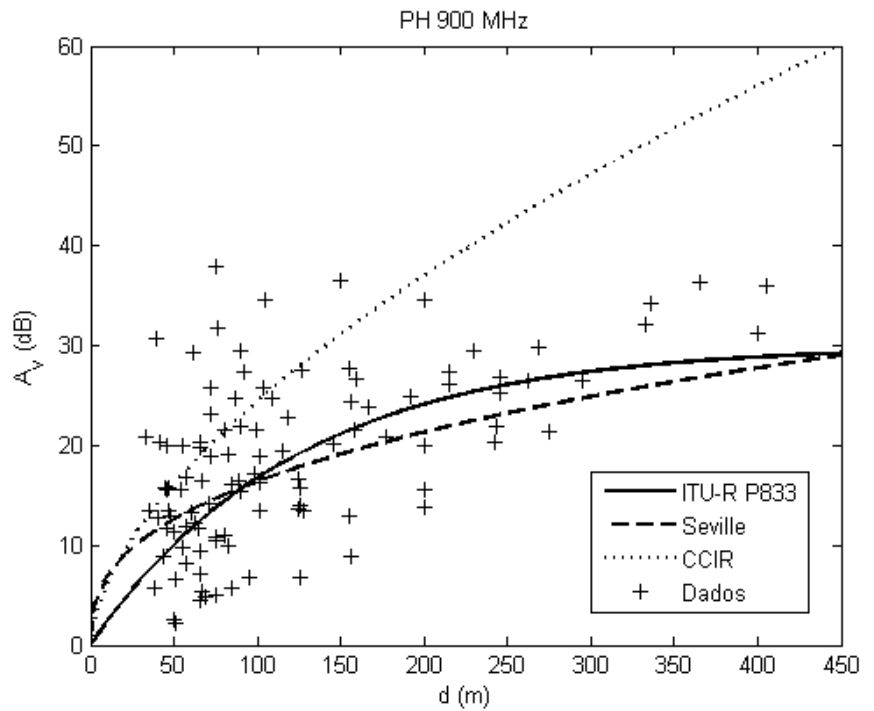

Fig. 4. Dados e modelos analisados em $900 \mathrm{MHz}$, polarização horizontal. 


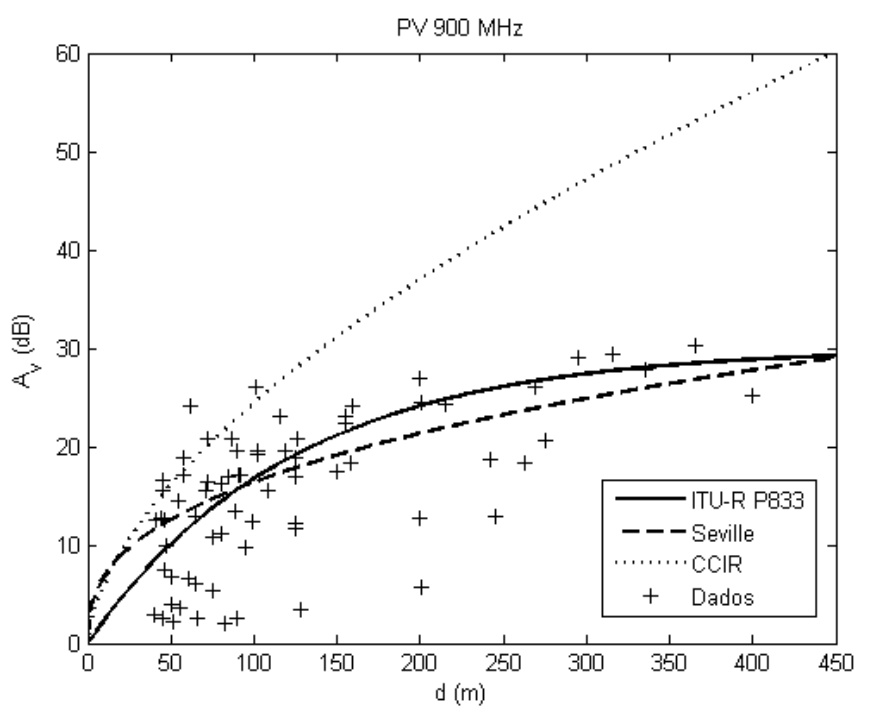

Fig. 5. Dados e modelos analisados em $900 \mathrm{MHz}$, polarização vertical.

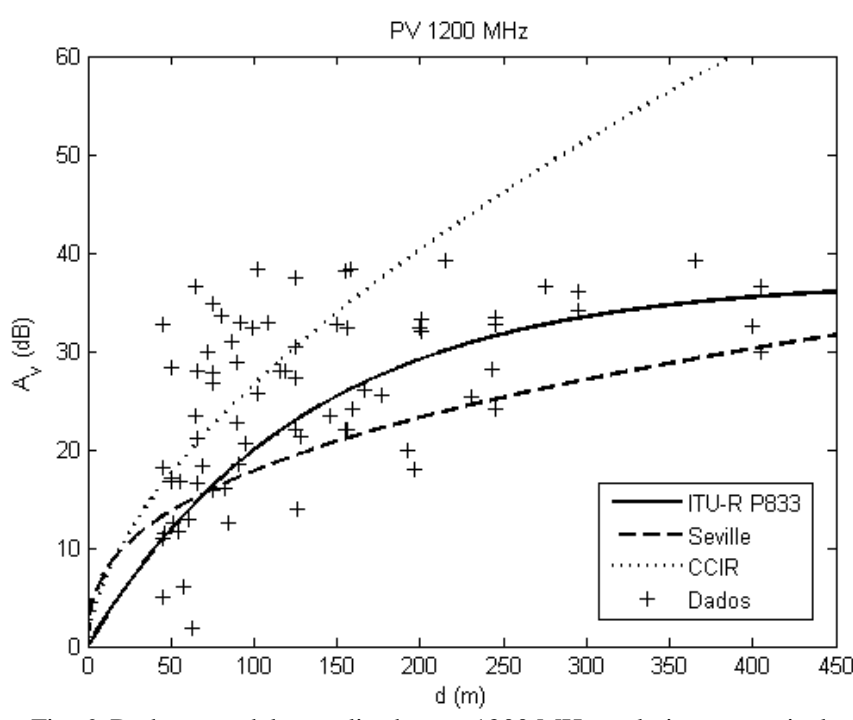

Fig. 6. Dados e modelos analisados em $1200 \mathrm{MHz}$, polarização vertical.

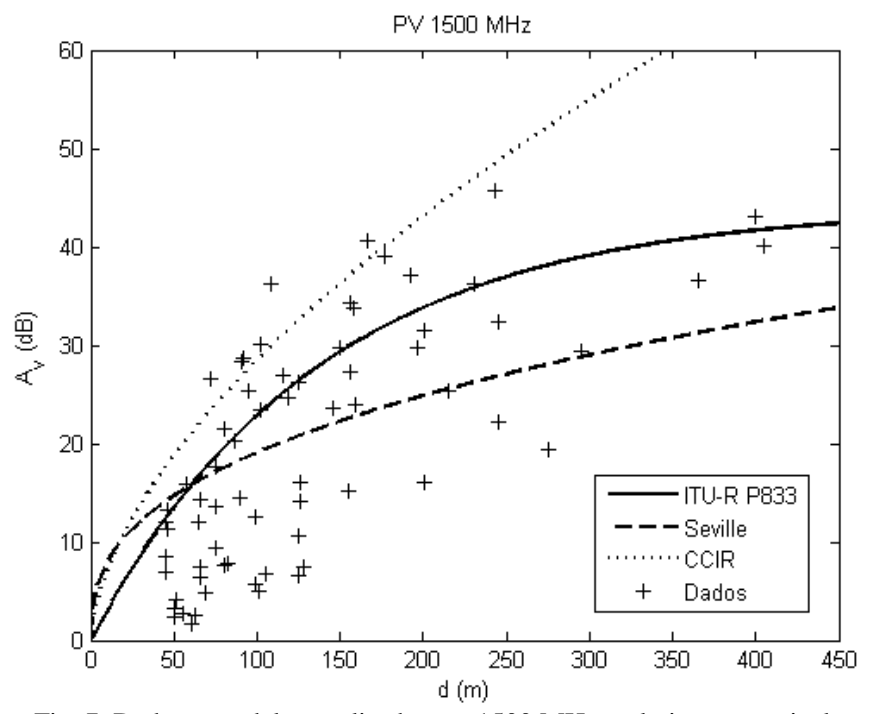

Fig. 7. Dados e modelos analisados em $1500 \mathrm{MHz}$, polarização vertical.

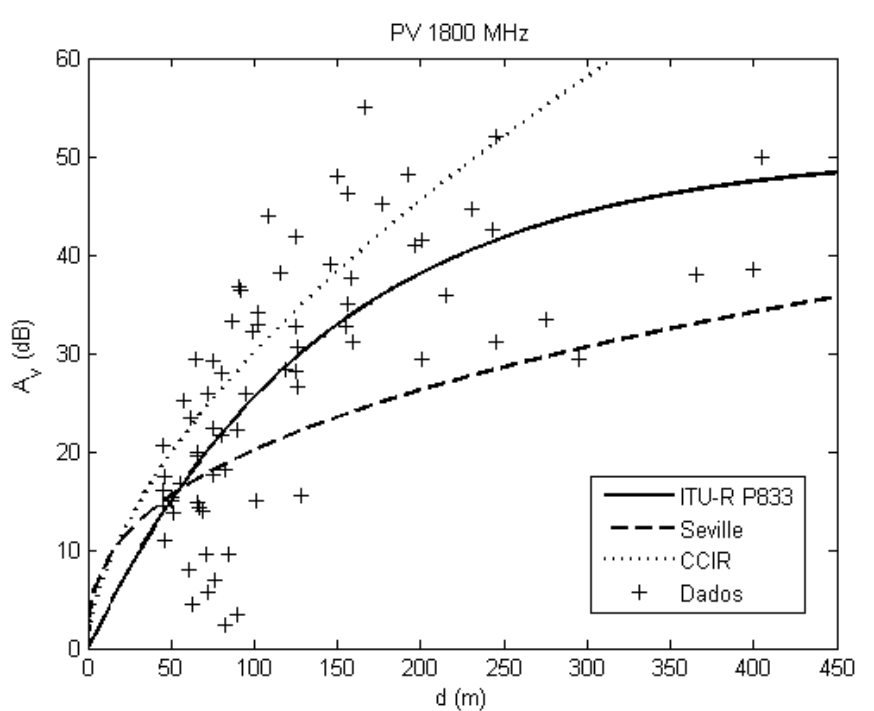

Fig. 8. Dados e modelos analisados em $1800 \mathrm{MHz}$, polarização vertical.

TABELA II

ERROS MÉDIOS (DB) ENTRE OS DADOS E OS MODELOS ANALISADOS

\begin{tabular}{|c|c|c|c|c|c|}
\hline Modelo & PH900 & PV900 & PV1200 & PV1500 & PV1800 \\
\hline ITU-R P.833 & 2,0 & $-0,9$ & 3,8 & $-5,0$ & 0,4 \\
Seville & 2,0 & $-0,8$ & 6,2 & $-0,7$ & 6,2 \\
CCIR & $-7,2$ & $-10,8$ & $-5,2$ & $-12,5$ & $-5,7$ \\
\hline
\end{tabular}

TABELA III

DESVIOS-PADRÃO (DB) ENTRE OS DADOS E OS MODELOS ANALISADOS

\begin{tabular}{|c|c|c|c|c|c|}
\hline Modelo & PH900 & PV900 & PV1200 & PV1500 & PV1800 \\
\hline ITU-R P.833 & 7,6 & 6,7 & 8,6 & 9,5 & 8,8 \\
Seville & 7,4 & 6,6 & 9,7 & 9,2 & 11,2 \\
CCIR & 11,5 & 13,8 & 11,5 & 15,3 & 11,5 \\
\hline
\end{tabular}

Verifica-se que o antigo modelo do ex-CCIR não aderiu bem aos dados, em concordância com as observações de análises similares [17], [22]. Curiosamente, o modelo de Seville apresentou aderência tão boa quanto o próprio modelo ajustado aqui proposto, apesar de ter sido obtido em condições bastante diversas: em $38 \mathrm{GHz}$; em bosques com árvores de clima temperado; e para distâncias de até $45 \mathrm{~m}$. A faixa de distâncias cerca de $10 \times$ menor que a do presente trabalho e a maior concentração de umidade da vegetação tropical parecem ter compensado, de alguma forma, a grande diferença entre as frequências de operação dos dois trabalhos. Além disso, a maior parte dos dados está concentrada no primeiro ponto em que as curvas do modelo proposto e do modelo de Seville se interceptam, ou seja, na região do plano onde os modelos são mais parecidos. É provável que com um volume maior de medidas em distâncias efetivas maiores, além da segunda interseção, a divergência entre os modelos fique mais evidente, com maior aderência para o modelo na forma proposta.

\section{CONCLUSÃO}

Este trabalho apresentou um modelo de perda adicional por vegetação em bosques urbanos, resultante do ajuste de medidas experimentais ao modelo geral da Recomendação P.833 do UIT-R. Os dados foram obtidos a partir de uma campanha de medidas realizadas no Campo de Santana, um parque arborizado no Centro do Rio de Janeiro, em quatro 
frequências representativas da faixa de operação de sistemas de comunicações móveis. Embora composto por diversas espécies de árvores, o bosque apresenta predominância da espécie Ficus Religiosa, bastante comum na cidade, e que atinge, em média, alturas de $15 \mathrm{~m}$.

Uma das motivações do trabalho realizado foi contribuir com parâmetros para a P.833, que indica a forma geral e apresenta valores típicos de atenuação específica, mas não traz valores gerais de outro parâmetro necessário, a atenuação máxima para um terminal dentro de uma floresta específica. As expressões deste parâmetro resultantes do presente trabalho foram aceitas como contribuição para a referida recomendação, e constam da sua última revisão [18].

O modelo obtido foi comparado a outros modelos disponíveis na literatura, para fins de avaliação. Um antigo modelo do ex-CCIR não apresentou boa aderência aos dados, como esperado. Por outro lado, um modelo similar proposto em [17] aderiu quase tão bem aos dados quanto o próprio modelo aqui proposto, o que não necessariamente deve se repetir para conjuntos de dados obtidos em distâncias efetivas maiores que as deste trabalho.

\section{AGRADECIMENTOS}

Os autores agradecem aos alunos e professores da PUC-Rio (CETUC) e da UFF (Departamento de Telecomunicações) que também participaram direta ou indiretamente da campanha de medidas, e à Fundação Parques e Jardins da cidade do Rio de Janeiro por permitir a realização do trabalho no Campo de Santana.

\section{REFERÊNCIAS}

[1] T. S. Rappaport, Wireless Communications: Principles and Practice, $2^{\text {nd }}$ Ed, Prentice-Hall, 2001

[2] M. Hata, "Empirical formula for propagation loss in land mobile radio services", IEEE Transactions on Vehicular Technology, vol. 29, no. 3, pp. 317-325, Ago 1980.

[3] H. L. Bertoni, Radio Propagation for Modern Wireless Systems, Prentice-Hall, 2000.

[4] T. K. Sarkar, Z. Ji, K. Kim, A. Modouri, M. S.-Palma, "A Survey of Various Propagation Models for Mobile Communication", IEEE Antennas and Propagation Magazine, vol. 45, no. 3, pp. 51-82, Jun 2003.

[5] M. H. C. Dias, Análise Crítica da Propagação em Microcélulas, dissertação de Mestrado, Instituto Militar de Engenharia, Rio de Janeiro - RJ, Jan 1998.

[6] L. L. Foldy, "The Multiple Scattering of Waves", Physical Review, vol. 67, no. 3-4, pp. 107-119, Fev 1945.

[7] M. Lax, "Multiple Scattering Waves", Rev. Mod. Phys., vol. 23, no. 4, pp. 287-310, 1951

[8] V. Twersky, "Multiple Scattering of Eletromagnetic Waves by Arbitrary Configurations", J. Math. Phys., vol. 8, no. 3, pp. 589-610, 1967.

[9] S. A. Torrico, H. L. Bertoni, R. H. Lang, "Modelling Tree Effects on Path Loss in a Residential Environment", IEEE Transactions on Antennas and Propagation, vol. 46, no. 6, pp. 872-880, Jun 1998.

[10] G. S. Brown, W. J. Curry, "A theory and model for wave propagation through foliage", Radio Science, vol. 17, no. 5, pp. 1027-1036, Set/Out 1982.

[11] S. S. Seker, "Multicomponents discrete propagation model of forest", IEE Proc. Microw. Antennas Propag., vol. 142, no. 3, pp. 201-206, Jun 1995.

[12] M. O. Al-Nuaimi, A. M. Hammoudeh, "Measurements and predictions of attenuation and scatter of microwave signals by trees", IEE Proc. Microw. Antennas Propag., vol. 141, no. 2, pp. 70-76, Abr 1994.
[13] J. Richter, R. F. S. Caldeirinha, M. O. Al-Nuaimi, A. Seville, N. C. Rogers, N. Savage, "A generic narrowband model for radiowave propagation through vegetation", in $61^{\text {st }}$ IEEE Vehicular Technology Conference - VTC 2005-Spring, Estocolmo - Suécia, vol. 1, pp. 39-43, Mai/Jun 2005.

[14] W. J. Vogel, J. Goldhirsh, "Tree Attenuation at $869 \mathrm{MHz}$ Derived from Remotely Piloted Aircraft Measurements", IEEE Transactions on Antennas and Propagation, vol. AP-34, no. 12, pp. 1460-1464, Dez 1986.

[15] K. Benzaïr, "Measurements and modelling of propagation losses through vegetation at 1-4 GHz", in $9^{\text {th }}$ International Conference on Antennas and Propagation, Eindhoven - Holanda, vol. 2, pp. 54-59, Abr 1995.

[16] S. S. Seker, A. Schneider, "Experimental characterisation of UHF radiowave propagation through forests", IEE Proceedings $H$ Microwaves, Antennas and Propagation, vol. 140, no. 5, pp. 329-335, Out 1993.

[17] A. Seville, U. Yilmaz, P. G. V. Charriere, N. Powell, K. H. Craig, "Building scatter and vegetation attenuation measurements at $38 \mathrm{GHz}$ ", in $9^{\text {th }}$ International Conference on Antennas and Propagation, Eindhoven - Holanda, vol. 2, pp. 46-50, Abr 1995.

[18] Attenuation in Vegetation, Rec. UIT-R P.833-6, Genebra, 2007.

[19] E. B. Atutov, Y.. L. Lomukhin, "Average Field in a Forest Medium", Journal of Communications Technology and Electronics, vol. 52, no. 11, pp. 1259-1264, 2007.

[20] Influences of terrain irregularities and vegetation on tropospheric propagation, CCIR Report 236-6, Genebra, 1986.

[21] J. C. R. Dal Bello, G. L. Siqueira, and H. L. Bertoni, "Theoretical Analysis and Measurement Results of Vegetation Effects on Path Loss for Mobile Cellular Communication Systems", IEEE Transactions on Vehicular Technology, vol. 49, no. 4, pp. 1285-1293, Jul 2000.

[22] A. Seville, K. H. Craig, "Semi-empirical model for millimetre-wave vegetation attenuation rates", Electronics Letters, vol. 31, no. 17, pp. 1507-1508, Ago 1995. 